\title{
Erratum to: Persistence and dioxin-like toxicity of carbazole and chlorocarbazoles in soil
}

\author{
John Mumbo • Bernhard Henkelmann • Ahmed Abdelaziz • Gerd Pfister • \\ Nghia Nguyen • Reiner Schroll • Jean Charles Munch • Karl-Werner Schramm
}

Published online: 25 September 2014

(C) Springer-Verlag Berlin Heidelberg 2014

\section{Erratum to: Environ Sci Pollut Res DOI 10.1007/s11356-014-3386-6}

The following errors have been detected in sections of the article outlined below. They have no influence on the results. Introduction

In the second paragraph, "and" is deleted from the sentence "Carbazole and 3,4,5,6-dibenzocarbazole are carcinogenic with the latter and also mutagenic (Lauby-Secretan et al. 2011)".

The sentence now reads;

"Carbazole and 3,4,5,6-dibenzocarbazole are carcinogenic with the latter also mutagenic (Lauby-Secretan et al. 2011)". In the last paragraph, "and" is also deleted from the sentence "Since according to the European Chemical Agency, a compound with a half-life of $>120$ days in soil is considered persistent (ECHA 2012), and a longer study period was adopted".

The sentence now reads;

The online version of the original article can be found at: http:// dx.doi.org/10.1007/s11356-014-3386-6.

J. Mumbo • B. Henkelmann • A. Abdelaziz • G. Pfister •

K.-W. Schramm $(\square)$

German Research Center for Environmental Health, Molecular

EXposomics (MEX), Helmholtz Zentrum München, Ingolstädter

Landstraße 1, 85764 Neuherberg, Germany

e-mail: schramm@helmholtz-muenchen.de

J. Mumbo $\cdot$ A. Abdelaziz $\cdot$ K.-W. Schramm

Department für Biowissenschaftliche Grundlagen, Technische

Universität München, Weihenstephaner Steig 23, 85350 Freising,

Germany

N. Nguyen $\cdot$ R. Schroll • J. C. Munch

German Research Center for Environmental Health, Institute of Soil

Ecology (ISE), Helmholtz Zentrum München, Ingolstädter

Landstraße 1, 85764 Neuherberg, Germany
"Since according to the European Chemical Agency, a compound with a half-life of $>120$ days in soil is considered persistent (ECHA 2012), a longer study period was adopted" Materials and methods

EROD induction bioassay and REP calculations

In the last paragraph of this section, "equivalent concentrations" is replaced with "toxic equivalent" in the sentence "Dioxin-like toxicity was then evaluated based on relative potency (REP) values with respect to 2,3,7,8-TCDD equivalent concentrations (TEQ $\left.{ }_{T C D D}\right)$ ".

The sentence now reads;

"Dioxin-like toxicity was then evaluated based on relative potency (REP) values with respect to $2,3,7,8$-TCDD toxic equivalent (TEQ $\left.{ }_{\mathrm{TCDD}}\right)$ ".

Criteria for compounds selection

The first sentence that reads "Monocarbazole, dicarbazole, tricarbazole and tetrabromocarbazole, chlorocarbazole and iodocarbazole were assessed in addition to $\mathbf{3 - m o n o c a r b a z o l e}-$ and 3,6-dichlorocarbazole studied previously (Tröbs et al. 2011)." has been corrected.

It now reads;

"Mono-, di-, tri- and tetra- substituted congeners of bromocarbazole, chlorocarbazole and iodocarbazole were assessed in addition to 3-chlorocarbazole- and 3,6dichlorocarbazole studied previously (Tröbs et al. 2011)."

Method validation

The first sentence in this section which reads "Each soil sample was then taken through all the steps of the methodology as outlined in $\underline{\mathbf{2 . 7} \text { and 2.10. }}$. has been corrected. The bolded and underlined words have been deleted and replaced with the right information.

It now reads;

"Each soil sample was then taken through the steps in the methodology as outlined under Sample collection and extraction, Cleanup and GC/MS analysis and quantification."

Results and discussions 


\section{Isolation, identification and concentration}

In the second paragraph of this section, the sentence that reads "However to our knowledge, the linkage between the $\underline{3}-$ $\underline{\text { monoisomer and 3,6-chloro isomer in the environmental }}$ samples and anthropogenic sources has not been demonstrated." has been corrected.

It now reads;

"However to our knowledge, the linkage between the 3mono- and 3,6-disubstituted chlorocarbazole isomers in the environmental samples and anthropogenic sources has not been demonstrated."

Dioxin-like toxicity assessment

"s" and "d" have been deleted, and "3,6-dibromocarbazole" has been replaced with "3,6-dichlorocarbazole" in the third sentence of the caption of Table 1 which reads "Pure compounds of 3-chlorocarbazoles and 3,6-dibromocarbazoles were used to calculate their respective REPs which were then used to calculate TEQ values for each soil extract".

It has been corrected to read;

"Pure compounds of 3-chlorocarbazole and 3,6dichlorocarbazole were used to calculate their respective REPs which were then used to calculate TEQ values for each soil extract".

In the second paragraph "3-Monocarbazole" is replaced with "3-Chlorocarbazole" in the sentence that reads "3Monocarbazole- and 3,6-dichlorocarbazole were only used in $\sum$ TEQ calculations making our results not conclusive .........".

It has been corrected to read;

"3-Chlorocarbazole and 3,6-dichlorocarbazole were only used in $\sum$ TEQ calculations making our results not conclusive to show this correlation but useful in predicting toxicity pattern for emerging category of environmental contaminants."

The last sentence of the caption in Table 2 that reads "For nonbinding molecules, a flag is given, where $(\mathrm{H})$ indicates that none of the identified poses features a sufficiently high hydrogen-bonding saturation and (S) indicates that none of the identified poses features a sufficiently high hydrophobic saturation nor hydrogen-bonding saturation." has been corrected.

It now reads;

"For non-binding molecules, a flag is given, where $(\mathrm{H})$ indicates that none of the identified possesses features of a sufficiently high hydrogen-bonding saturation and (S) indicates that none of the identified possesses features of a sufficiently high hydrophobic saturation or hydrogen-bonding saturation".

Implications of halogenated carbazoles in environment

In the last paragraph, the sentence that reads "9-Butyl-9HCarbazole has been classified as high priority PBT substance (Brooke and Burns 2010)." has been corrected.

It now reads;

"9-Butyl-9H-Carbazole has been categorized as high priority PBT substance for further investigations (Brooke and Burns 2010)."

\section{Conclusions}

The first and second last sentences that read "Carbazole, $\underline{3-}$ monocarbazole and 3,6-dichlorocarbazole are persistent to degradation in soil." and "3,6-Dibromocarbazoles, $\underline{\mathbf{3}}$ monocarbazoles and 3,6-dichlorocarbazoles possess dioxinlike toxicity." have been corrected to remove the errors.

The conclusion now reads,

"Carbazole, 3-chlorocarbazole and 3,6-dichlorocarbazole are persistent to degradation in soil. 3,6-Dichlorocarbazole was the most resistant under the two temperature conditions. This was probably due to the halogenation effect and hence the consistently high concentrations of 3,6-dichlorocarbazole recorded in all soil samples relative to other compounds. 3,6Dibromocarbazole, 3-chlorocarbazole and 3,6dichlorocarbazole possess dioxin-like toxicity. They are potential POP with the possibility of being rated in the category of PBT substances similar to carbazole." 